\title{
Research on Fuzzy Control Method in EDM Machine
}

\author{
Haiyan Wang, Baiyu and Xuejun Li \\ Chang chun university of science and technology \\ Chang chun university of technology \\ Chang chun university \\ 609872165@qq.com,13742883@qq.com,524625282@qq.com \\ Abstract \\ Processing effect is a lot of. In this paper, the research perspective is to-improve the \\ machining efficiency and machining quality of the system. Bosed on the analysis of the \\ characteristics of the edm process as the foundation, proposed a fuzzy control technology. In \\ edm process, using fuzzy neural network control technology. According to the characteristics \\ of the fuzzy controller, combined with the advantages of artificial neural network, to correct \\ the adverse effect of some imperfect rules, improve the fast response ability of the control
} system in a reliable way.

Keywords: EDM; fuzzy neural control, furzy controller, Artificial Neural Networks;

\section{Introduction}

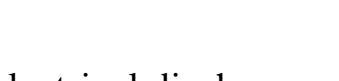

The physical process of electrical discharge machining is very short and complicated, each of the micro proces of spark errosion is the electric field force, electromagnetic force, thermodynamic fluid power electrochemical and colloid chemistry and so on synthesis process. Now for this process to establish a precise mathematical model is impossible. For this problem, a better approach is to use the fuzzy control, use of some characteristics of its mathematical model is expressed, establish fuzzy control rules, and expressed in the form of fuzy language. When the control process is too complex, with a simple fuzzy control system is difficult to establish effective decision rules. Using artificial neural network can just make up for the inadequacy of fuzzy system in this regard. . The combination of fuzzy system and artificial neural network is constituted fuzzy neural control system, this is the one with adaptive system of human sensory and cognitive components, the neural network directly embedded in a full fuzzy and neural network structure, In the structure of fuzzy training data to learn, produce, and highly summarized correction between input and output fuzzy rules. Then, according to the geometrie distribution of input fuzzy set and the fuzzy rules produced by past experience, they can get from this reasoning to the correct conclusion.

\section{The fuzzy control throry}

Application of fuzzy logic in control field are called fuzzy control. Fuzzy control is one of the biggest characteristics is that it will be the operator or expert control experience and knowledge representation language variables to describe the control rules, and then use these rules to control the system. Therefore, fuzzy control mathematical model is especially suitable for the unknown and complicated nonlinear 
system control. From the perspective of information, fuzzy control is a kind of rulebased expert system, it is also a class of nonlinear controller.

For each language rules in the fuzzy controller, Like: "if $\mathrm{A}$ is $\mathrm{Ak}$ and $\mathrm{B}$ is $\mathrm{Bk}, \mathrm{C}$ as $\mathrm{Ck}$, Defines a fuzzy relation contains the type (Ak, Bk Ck). the input value to one or more Ak and Bk fuzzy rules, which based on fuzzy reasoning to determine output fuzzy subset, real output is obtained by solving fuzzy process again, Real output is obtained by fuzzy process again. On the theory of the specified domain, all rules are defined the se vague relationship, form a kind of mapping fF, as shown in Figure 1. Due to the uncertainties of the mapping relationship itself, difficult to express with mathematics type.

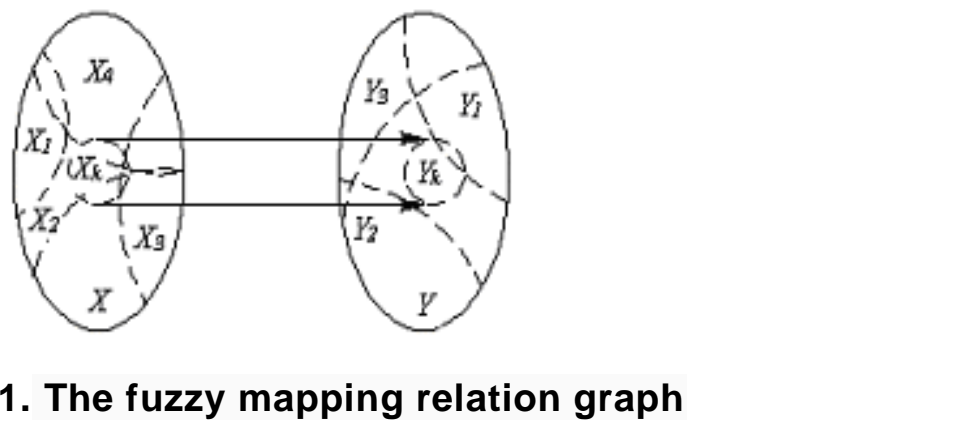

Figure 1. The fuzzy mapping relation graph

Schematic is shown in Figure 2 is the fuzzy control system, the control system of the input and output are exact value, and the control algonithm is the fuzzy algorithm. The basic component of the fuzzy controller mainly contans fuzzification interface, rules library, fuzzy reasoning, narrated the interfacesection. Process the input of the fuzzy control system is difference of the measured variables and system Settings (e), and the deviation of the rate of change (ec). Output is a system of real-time control variables (u), the input of precise quantity through fuzzy sets are into E and C. Fuzzy multiplier is the core of fuzzy controher, it will be the person's experiences, thinking process, summarized into fuzzy relation and fuzzy reasoning rules, fuzzy reasoning is a kind of fuzzy transformation, it will transform input variable fuzzy sets for the output variable fuzzy sets, and realize the transformation of domain, $U$ get output fuzzy sets, fuzzy judgment after fuzzy clear nto a precise amount, to control the controlled object.

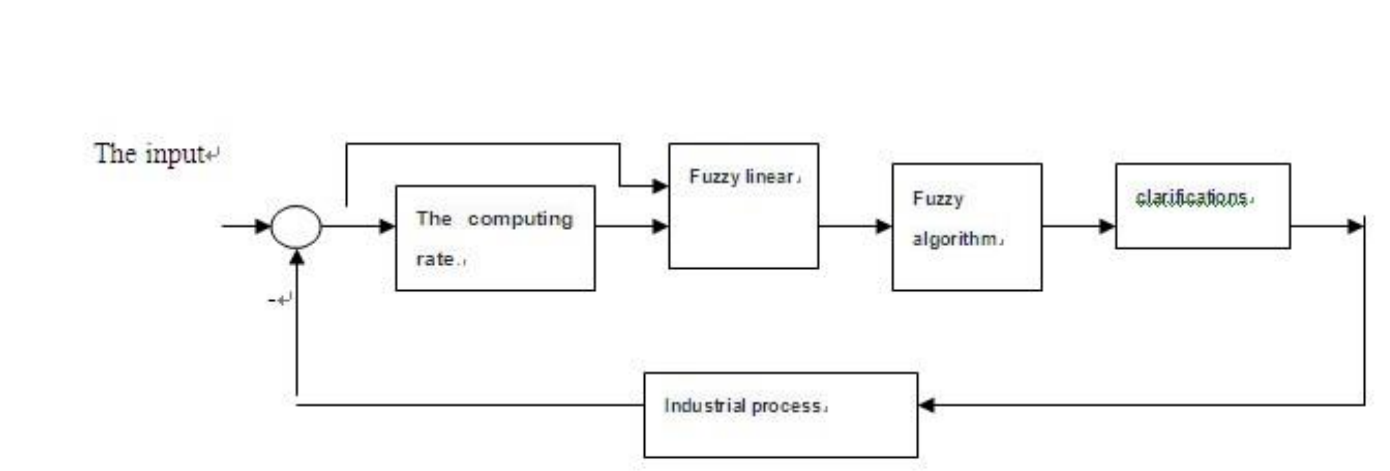

Figure 2. Fuzzy control system diagram 


\section{The structure of the artificial neuron}

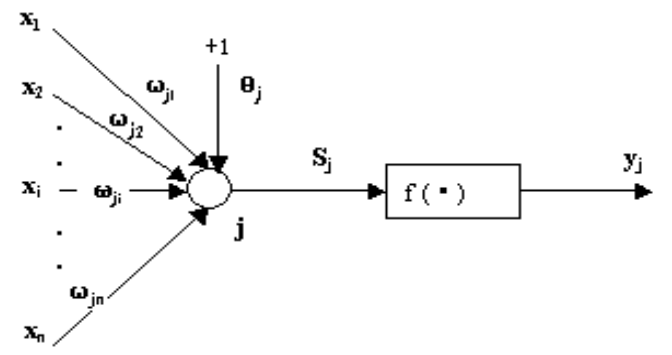

\section{Figure 3. The structure of the artificial neuron}

Shown in figure 3 are modelled on the basic characteristics of biofogical neuron structure of typical artificial neuron model.

$x_{1}, x_{2}, \ldots, x_{i}, \ldots, x_{n}$ representing the input from other neurons.

$\omega_{j 1}, \omega_{j 2}, \ldots, \omega_{j i}, \ldots, \omega_{j n}$ respectively according to neurons $1,2, \ldots, \mathrm{i}, \ldots, \mathrm{n}$ With the

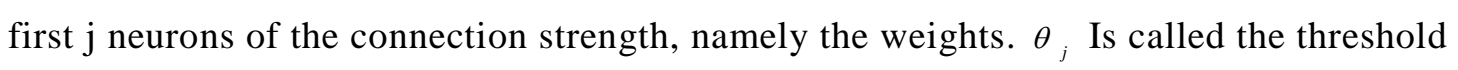
also known as the threshold, generally it is a fixed offset +

Net input of neurons $S_{j}$ through the transfer function $f(\bullet)$, output of the neuron is:

\section{BP algorithm}

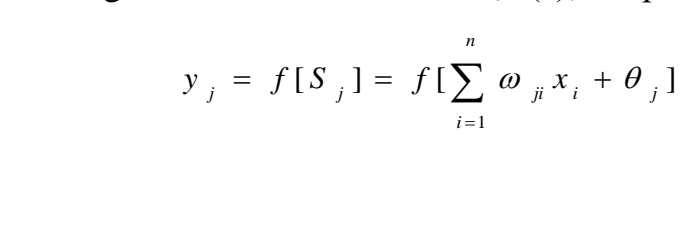

Neural network to spread the information stored in the form of weight matrix in the training process of neural networks is the right of matrix modification process, make it comply with the needs of the research problem. As a result of the single layer neural network can only Solve linearly separable problems, even the simple "xor problem can not solve, in this paper, ingrder to have a hidden layer of three layer feed forward network as an example, analyzes its Error backward Propagation algorithm (the Error Back Propagation, BP algorithm) modify the principle of weight matrix. A single hidden layer feed forward network topology structure as shown in Figure 4:

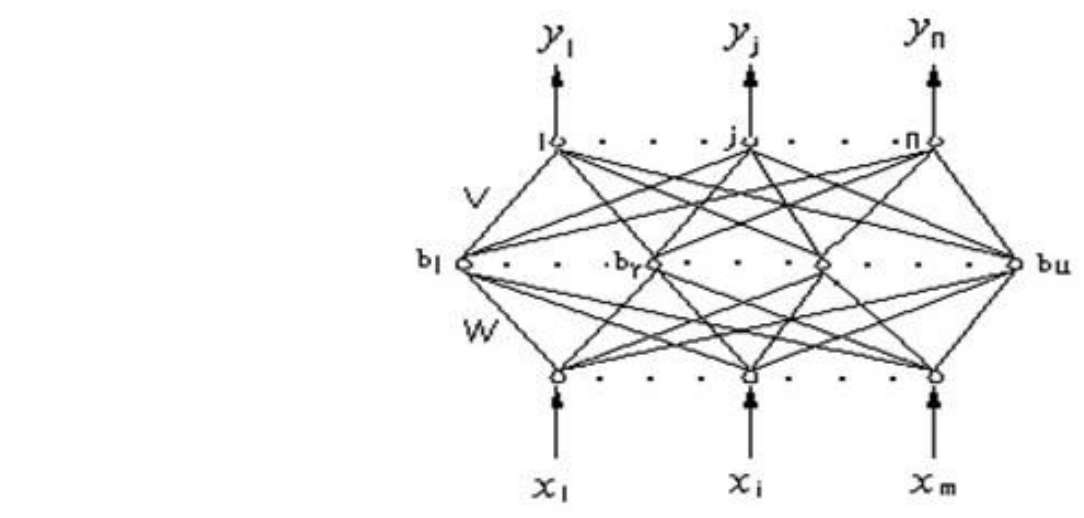

Figure 4. A single hidden layer feed forward network topology 
Set input layer nodes $x_{i}$ to the hidden layer nodes $b_{r}$ connection has a weight of $\omega_{i r}$ , of hidden layer nodes $b_{r}$ to the output layer nodes $y_{j}$ connection has a weight of $v_{r j}$ , $T_{r}$ as the input layer node threshold, $\theta_{j}$ for the output layer node threshold, Is the BP algorithm training rule is as follows:

(1) The preset a small random weight matrix $\mathrm{W}, \mathrm{V}$.

(2) For an input mode: $x_{1}, x_{2}, \ldots, x_{i}, \ldots, x_{n}$, according to the formula (1), the output layer nodes in turn forward calculation:

$$
\begin{aligned}
& b_{r}=f\left(\sum_{i=1}^{m} \omega_{i r} \cdot x_{i}+T_{r}\right) \quad(r=1, \cdots, u) \\
& y_{j}=f\left(\sum_{r=1}^{u} v_{r j} \cdot b_{r}+\theta_{j}\right) \quad(j=1, \cdots, n)
\end{aligned}
$$

(3) Calculate the output layer, and the first $\mathrm{j}$ a node connected weights $v_{\text {ir }}$ modifier $\Delta v_{j r}$, set $y_{j}$ the ideal output for $t_{j}$, a transfer function for single polarity Sigmoidfunction : $y=f(s)=\frac{1}{1+e^{-s}}$, then

$$
\begin{aligned}
& f^{\prime}(s)=(1-y) y \\
& \Delta v_{j r}=-\alpha \frac{\partial E_{p}}{\partial v_{j r}}=\alpha\left(t_{j}-y_{j}\right) f^{\prime}(s) b_{r}=\alpha\left(y_{j}\right) y_{j}\left(y_{j}\right) b_{r}=\alpha \delta_{y j} b_{r}
\end{aligned}
$$

In the formula: $\alpha$ as the vector $0<\alpha<1 E_{p}$ is to define the error function $E_{p}=\frac{1}{2} \sum_{j=1}^{n}\left(t_{j}-y_{j}\right)^{2}$

(4) Back to the hidden layer nodes distribution error:

$$
\begin{aligned}
\Delta \omega_{r i} & =-\beta \frac{\partial E}{\partial \omega_{r i}}=-\beta \frac{\partial E p}{\partial b} \frac{\partial b}{\partial s_{r}}=\beta\left[\sum_{j=1}^{n}\left(t_{j}-y_{j}\right) \frac{\partial y_{j}}{\partial s_{j}} \frac{\partial s_{j}}{\partial b_{r}}\right] \frac{\partial b_{r}}{\partial s_{r}} \frac{\partial s_{r}}{\partial \omega_{r i}} \\
& =\beta\left[\sum_{j=1}^{n} \delta_{y j} v_{j r} b_{j}\left(1-b_{r}\right) x_{i}=\beta \delta_{b r} x_{i}\right.
\end{aligned}
$$

In the formula: $\beta$ vector hidden layer $0<\beta<1 ; \delta_{b r}$ hidden layer error signal

$$
\left.\delta_{b r}=\sum_{i=1}^{n} \delta_{y j} v_{j r}\right] b_{r}\left(1-b_{r}\right)
$$

(5) Adjust the connection weights between nodes in each layer and node threshold:

$$
\begin{array}{rlr}
v_{j r}=v_{j r}+\alpha \delta_{j r} b_{r} & ; \quad \theta_{j}=\theta_{j}+\alpha \cdot \delta_{j r} \\
\omega_{r i}=\omega_{r i}+\beta \delta_{b r} x_{i} & ; & T_{r}=T_{r}+\beta \cdot \delta_{b_{r}}
\end{array}
$$

(6) Enter the next mode, continue to train the network, until the error function $E_{p}<E_{\max }$ so far, end of the study 。 


\section{The fuzzy neural controller}

In edm process, the introduction of fuzzy neural controller, can greatly improve the machining quality and efficiency of machine tool. EDM machine servo control system, mainly by the current feedback, fuzzy neural controller, servo system and EDM machine tool.

By adjusting the servo motor forward or backward, causes the gap distance between electrodes; according to the discharge electrodes and adjusting servo reference voltage, get the best discharge gap. System control block diagram as shown in figure 5. Use discharge gap average current,

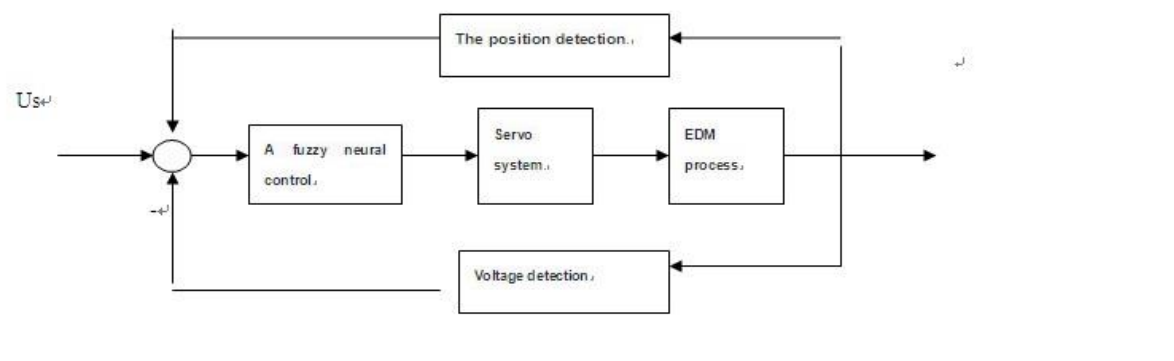

Figure 5. Edm machine servo control system block diagram

Represents the size of the gap between the electrode, through the setting of servo motor control parameter, to determine the distance between the poles. First through the input state detection circuit current aceutely detectearance on machining process, then compares it with best gap ayerage current, Get two input variables, clearance of current deviation $e(t)$ and the deviation $g$ hange rate, $e_{c}(t)$, the output is the adjustment of the drive system variation $\Delta i(t)$, then the three precise amount into fuzzy quantity. Accurate quantity $g(t)$ and the deviation change rate $e_{c}(t)$ set to $[-4+$ 4] change between continuous quantities, $\Delta i(t)$ set to $[-6+6]$ change between continuous quantities, can draw their membership degree curve :

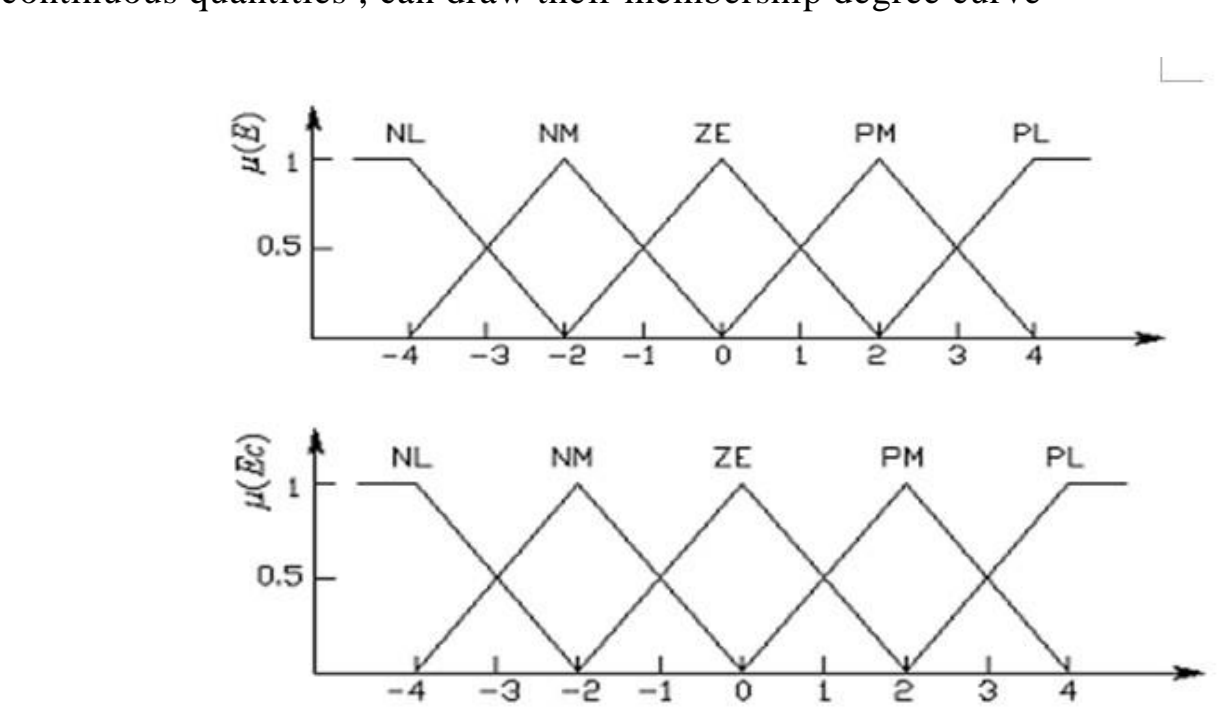




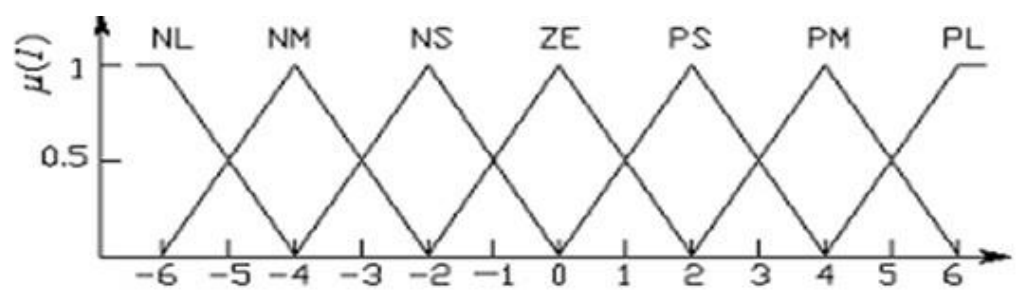

Determine $E(t), \quad E c(t)$ and $I(t)$ the fuzzy subsets are:

$$
\begin{aligned}
& \tilde{E}=\{P L, P M, Z E, N M, N L\} \\
& \tilde{E} c=\{P L, P M, Z E, N M, N L\} \\
& \tilde{I}=\{P L, P M, P S, Z E, N S, N M, N L\}
\end{aligned}
$$

In the neural network is shown in figure 4, each input unit corresponding to the input variable $E(t) 、 E c(t)$, a fuzzy subset, Network of the input signal format as follows, including $m=10$ nodes: as shown in figure 6, shown in Figure 7 .

$$
\left[\mu_{N L}(e), \mu_{N M}(e), \mu_{0}(e), \cdots, \mu_{0}(e c), \mu_{P M}(e c), \mu_{p}(e c)\right]
$$

Network output variable corresponding to each unit of output is a quantitative value of the theory of spatial domain, because the theory of servo reference current change quantity to $\Delta I$ domain is divided into 13 files, that is $\{6,-5,-4,-3,-2,-1,0$, $+1,+2,+3,+4,+5,+6\}$, The network output node number $n=13$, and its output signal format for: as shown in figure 8

$$
\left[\mu_{Y}\left(\Delta U_{s 1}\right), \mu_{Y}\left(\Delta U_{s 2}\right), \mu_{Y}\left(\Delta U_{s 3}\right), \cdots, \mu_{Y}\left(\Delta U_{s-2}, \mu_{Y}\left(\Delta U_{s n-1}\right), \mu_{Y}\left(\Delta U_{s n}\right)\right]\right.
$$

Before using the neural network, and it is initiatized to the learning and training, and input or adjust the fuzzy rules, and the introduction of fuzzy neural network controller.

\section{Conclusion}

Fuzzy neural control system is the combination of fuzzy control and neural network, and the research direction caught the attention of people. Fuzzy control and neural network does nôt need precise model of controlled object, a fuzzy neural network controller is usually composed of a neural network and fuzzy algorithm. Among them, the fuzzy controller using fuzzy inference rules, to simulate the processing of uncertainty in the progess of decision-making behavior, but to automatically generate rules from experience, and modify the self-learning function of the control decision making is also imperfect. The introduction of the neural network performance index in the process of fuzzy control is better, to make the fuzzy neural control system in the edm process in-depth research and extensive application, provides a great possibility

\section{Rererences}

[1] Z. Wansheng, "Technology of EDM [M]", Haerbin: Haerbin University of Technology Press, (2000).

[2] M. KangXiong, "Theory and technic of EDM [M]", Dongjing, (1972).

[3] F. Gang, "A New Stable Tracking Control Scheme for Robotic Manipulators", IEEE Trans Sys, Man, Cybem, vol. 27, no. 3, (1997), pp. 510-516.

[4] F. L. Lewis, "Neural Net Robot Controller with Guaranteed Tracking Perfomance, "IEEE Teans Neural Networks, vol. 6, no. 3, (1995), pp. 703-715.

[5] C. Gong, "Fuzzy neural network and its application in electrical discharge machining", [M], Control Theory and Control Engineering, (2005). 
[6] Jianhua, W. Hui and X. Qihua, "A fuzzy neural network based new method of edm processing conditions was determined [J]”, Machinery Science and Technology, vol. 08, (2008).

[7] C. Ming and G. Lin, Z. Shou and Wang, "Temperature superplastic forming technology based on fuzzy neural network parameters optimization [J]", Journal of Nanjing University of Aeronautics and Astronautics, vol. 04, (2005).

[8] X. Qihua, Jianhua, said ehud Shapiro, "Road intersection traffic control method based on fuzzy neural network [J]", Computer engineering, vol. 14 (2004).

[9] Liu, Z. Zongshu and Y. Y. Ice, "Fuzzy neural network based on subtractive clustering end temperature prediction model of converter [J]", Journal of materials and metallurgy, vol. 04, (2006).

[10] Z. Wansheng, "Edm technology [M]", Harbin industrial university press, (2000).

[11] S. K. Oh, "Rule -based multi - FNN identification with the aidof evolutionary fuzzy granulation", Knowledge - -based Systems, (2004).

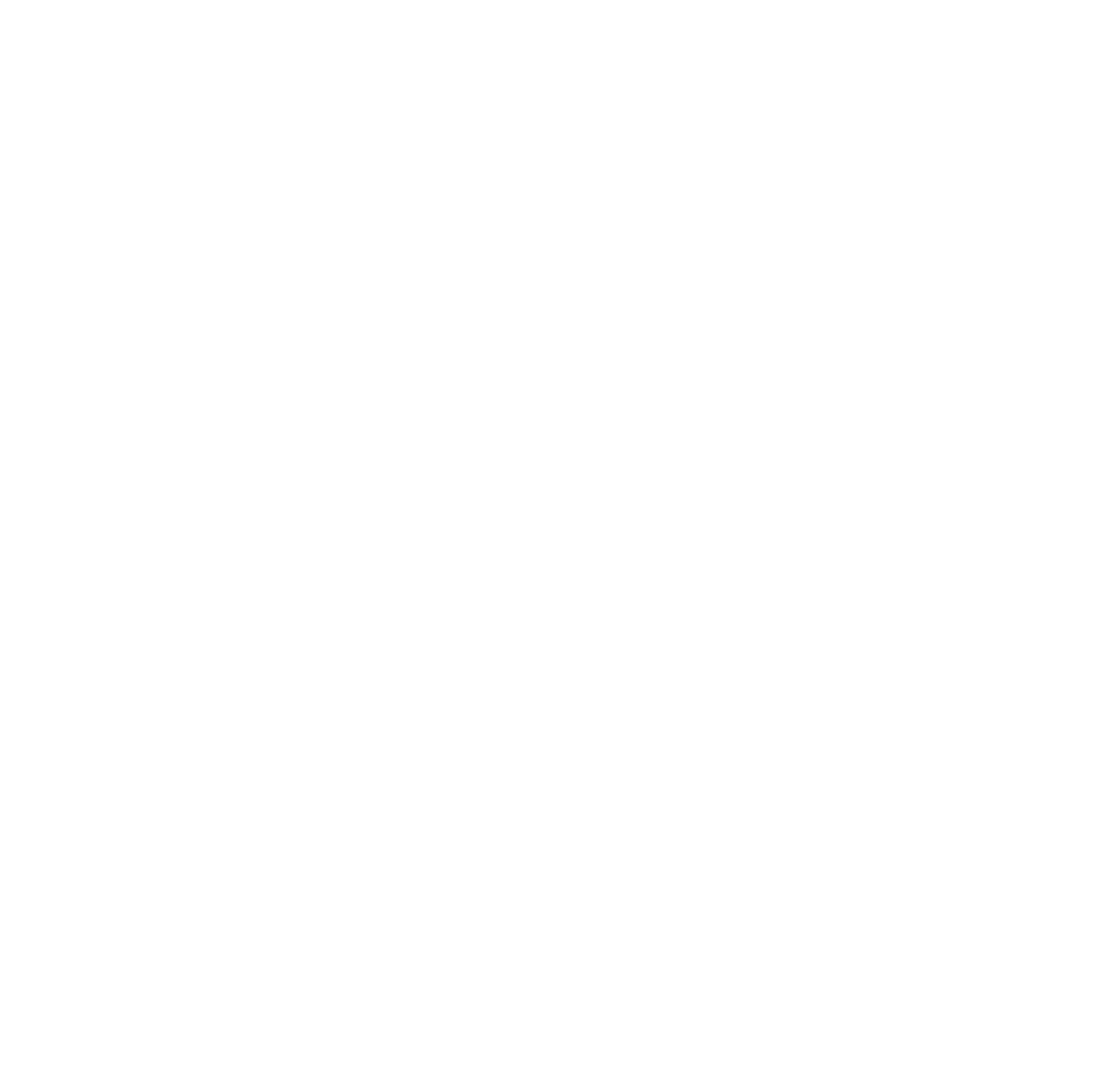


International Journal of Hybrid Information Technology

Vol.7, No.3 (2014)

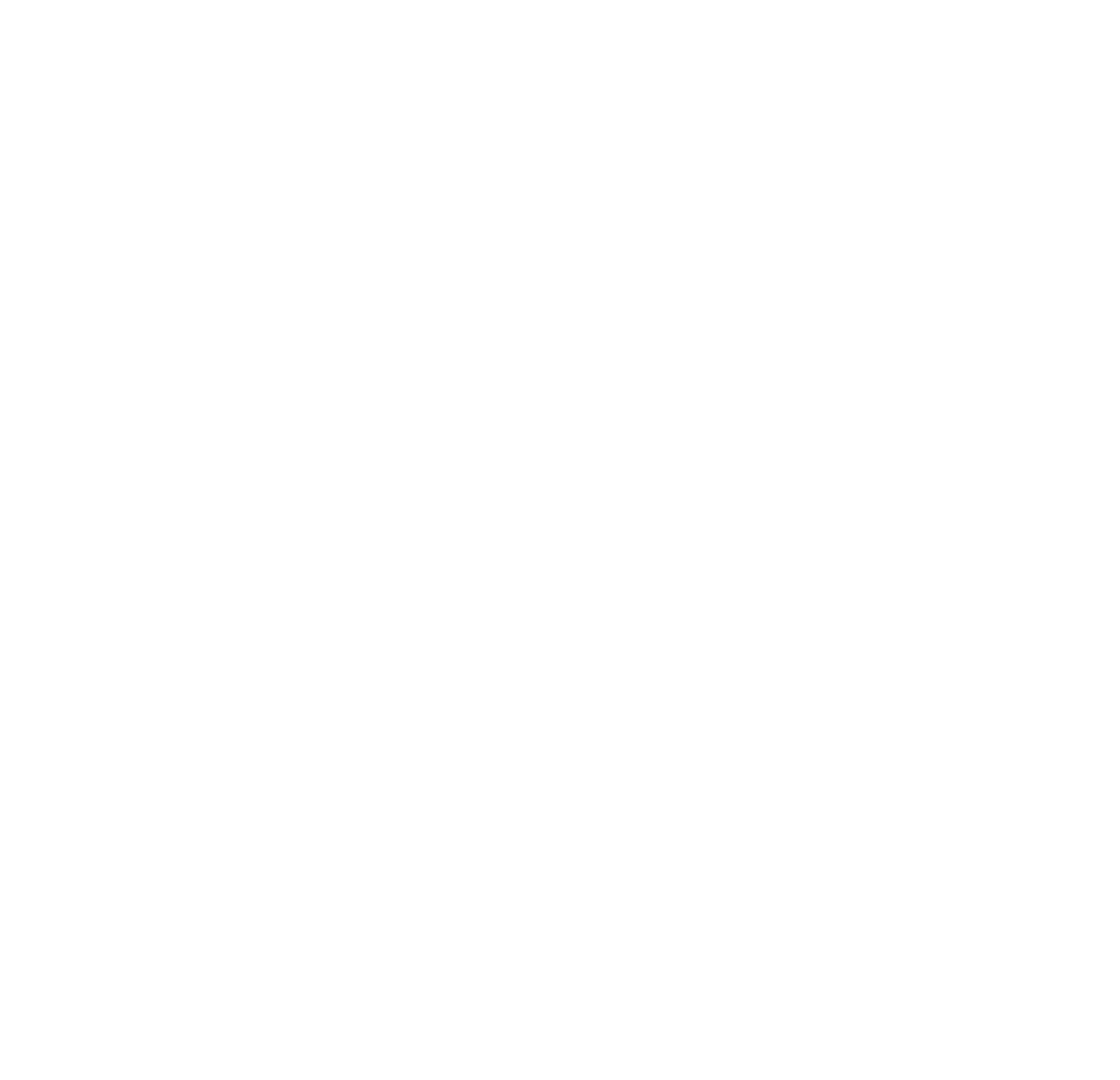

